\title{
ON THE SPECTRA OF PRESPECTRAL OPERATORS
}

by B. NAGY

(Received 14 July, 1976)

The spectrum of a prespectral operator was investigated by Dowson in [4]. The question was left open there whether, if a prespectral operator has closed range, the same is true for its scalar part. In this paper we answer this in the affirmative and point out some consequences concerning the essential spectra of prespectral operators. Also, following Taylor and Halberg [11], we present the state diagram of a prespectral operator, which will show, in a sense, the sharpness of the results of the spectral theory of such operators.

We will assume familiarity with the basic properties of prespectral operators given in [1], [3] and [4]. In the paper $X$ denotes a Banach space over the complex field $\mathbf{C}$, with norm $|\cdot|$ and dual space $X^{*} . B(X)$ denotes the Banach algebra of bounded linear operators on $X$. If $V$ is in $B(X), Z(V), R(V), s(V), r(V)$ and $V^{*}$ denote its null space, range, spectrum, resolvent set and adjoint operator, respectively. For a prespectral operator $T$, in $B(X)$, of class $\Gamma, T=S+N$ denotes its canonical decomposition and, as a rule, $E$ denotes its resolution of the identity of class $\Gamma$. $T \mid Y$ denotes the restriction of $T$ to the subspace $Y$, and if $z \in \mathbf{C}$, then $E_{z}, E_{z^{\prime}}$ and $N_{z}$ denote $E(\{z\}), E(C \backslash\{z\})$ and $N \mid E(\{z\}) X$, respectively. If $I$ is the ideritity in $B(X)$, we shall write $T-z$ rather than $T-z I$.

THEOREM 1. If $T$ is prespectral with closed range, then $R(S)$ is closed.

Proof. Let $E$ denote the resolution of the identity of class $\Gamma$ for $T$, and assume first that $E_{0}=0$. Then $Z(T)=\{0\}$ by [4, Theorem 6.13]. Since $R(T)$ is closed, there is a positive $p$ such that for every $x$ in $X$ we have $|T x| \geqslant p$ dist $(x, Z(T))=p|x|$. By a result of Dowson ([2, Theorem 1]), then $0 \in r(T)$; hence $0 \in r(S)$.

If $E_{0} \neq 0$, then the restriction $V=T \mid E_{0} X$ in $B\left(E_{0}, X\right)$ is prespectral with resolution of the identity $F(b)=E(b) \mid E_{0^{\prime}} X$ of class $\Gamma^{\prime}$ (here $\Gamma^{\prime}=\left\{g+\left(E_{0^{\prime}} X\right)^{\perp} ; g \in \Gamma\right\} \subset$ $X^{*} /\left(E_{0} \cdot X\right)^{\perp}$, where $H^{\perp}$ is the annihilator in $X^{*}$ of $\left.H \subset X\right)$, and $F_{0}=0$. If $y \in \overline{R(V)}$, then there is a sequence $\left\{x_{n}\right\}$ in $E_{0} X$ such that $V x_{n} \rightarrow y$, and $y=T x$, for some $x$ in $X$, because $R(T)$ is closed. Then $V E_{0} x=T E_{0^{\prime}} x=E_{0}, T x=y$; hence $R(V)$ is closed and, by the preceding paragraph, $0 \in r(V)$. From [3, Theorem 2] the scalar part of $V$ is $S \mid E_{0}, X$; thus $S E_{0^{\prime}} X=E_{0^{\prime}} X$, and $S E_{0}=0$ implies $S X=E_{0^{\prime}} X$; hence $R(S)$ is closed.

THEOREM 2. Suppose $T$ is prespectral with resolution of the identity $E$ of class $\Gamma$, and $z$ is a complex number. Then $R(T-z)$ is closed if and only if

(1) $z$ is an isolated point of $s(T)$ or is in $r(T)$, and

(2) $R\left((T-z) E_{z}\right)=R\left(N_{z}\right)$ is closed.

Proof. $T-z=S-z+N$, where $S-z$ is a scalar type prespectral operator with resolution of the identity $G(b)=E(b+z)$ (b Borel set) of class $\Gamma([1,3.1])$, so we may and shall suppose that $z=0$. The operator $T-c$ ( $c$ complex) is completely reduced by the subspaces $\left(E_{0} X ; E_{0^{\prime}} X\right)$ with restrictions $N_{0}-c E_{0}$ and $T \mid E_{0^{\prime}} X-c E_{0^{\prime}}$, respectively. If

Glasgow Math. J. 19 (1978) 57-61. 
$R(T)$ is closed, then the proof of Theorem 1 and [10, Section 5.4] yield that $(T-c)^{-1} \epsilon$ $B(X)$ for every $c$ in a punched neighbourhood of 0 ; thus (1) holds. Further, if $T E_{0} x_{n} \rightarrow y$, then $T x=y$ for some $x$ in $X$, for $R(T)$ is closed. But $T E_{0} x=E_{0} T x=E_{0} y=y$; thus (2) is also true.

Conversely, if (1) and (2) hold, $y \in \overline{R(T)}$ and $T x_{n} \rightarrow y$, then $T E_{0} x_{n} \rightarrow E_{0} y$; hence $T E_{0} v=E_{0} y$, for some $v$ in $X$. By (1), $E(U)=0$ for some punched open neighbourhood $U$ of 0 and, with the notation of Theorem $1, F_{0}=E_{0} \mid E_{0}, X=0$; therefore $F(U \cup\{0\})=0$. Hence $0 \in r\left(T \mid E_{0}, X\right)$; thus there is $u \in E_{0^{\prime}} X$ such that $T u=E_{0^{\prime}} y$. But then $T\left(E_{0} v+u\right)=y$, and the proof is complete.

For a linear operator $V$ in $B(X)$ the quantities nullity $n(V)$, defect $d(V)$, ascent $a(V)$ and descent $e(V)$ are defined e.g. in [7, p. 197]. We define the essential spectra, studied by Gramsch and Lay [6], by means of the regularity sets $G_{i}$, where $V \in G_{i}(i=1$, $2, \ldots, 11)$ means $G_{1}: V^{-1} \in B(X), G_{2}: n(V)=d(V)$ and $a(V)=e(V)$ are finite, $G_{3}: n(V)=d(V)$ are finite, $G_{4}: n(V)-d(V)$ is finite, $G_{5}: n(V)$ is finite and $R(V)$ is complemented, $G_{6}: d(V)$ is finite and $Z(V)$ is complemented, $G_{7}: n(V)$ is finite and $R(V)$ is closed, $G_{8}: d(V)$ is finite, $G_{9}=G_{7} \cup G_{8}, G_{10}: R(V)$ is closed, $G_{11}: a(V)$ and $e(V)$ are finite.

The essential spectrum $s_{i}(V)$ is the set of all complex numbers $c$ such that $V-c \notin G_{i}$ $(i=2,3, \ldots, 11)$. For $i=1$ we obtain the spectrum $s(V)$. The following result was obtained in [8] for spectral operators.

Lemma. If $T$ is a prespectral operator, then the essential spectra $s_{i}(T)(i=2,3, \ldots, 9)$ are identical.

Proof. If $z$ is a nonisolated point of $s(T)$, then Theorem 2 implies that $z \in s_{10}(T)$; hence $z \in s_{i}(T)(i=2,3, \ldots, 9)$. If $z$ is an isolated point of $s(T)$ and $T-z \in G_{9}$, then [7, Theorem 2.9] yields that $T-z \in G_{2}$. Since $G_{2} \subset G_{i} \subset G_{9}(i=3,4, \ldots, 8)$, the lemma is proved.

Theorem 3. If $T$ is in $B(X)$, then $s_{i}(T)=s_{i}\left(T^{*}\right)$ for $i=2,3,4,9,10,11, s_{7}(T)=s_{8}\left(T^{*}\right)$ and $s_{8}(T)=s_{7}\left(T^{*}\right)$. If, in addition, $T$ is prespectral, then $s_{i}(T)=s_{i}\left(T^{*}\right)$ for $i=2,3, \ldots, 11$.

Proof. Studying the various properties of $T-z$ and $T^{*}-z$ we may and shall suppose that $z=0$. Since $s\left(T^{*}\right)=s(T)$, their isolated points are also identical. For the resolvent operators, we have $R\left(u ; T^{*}\right)=R(u ; T)^{*}$; hence if 0 is an isolated singularity of $s(T)$ with Laurent expansion around 0 given by $R(u ; T)=\sum_{k=-\infty}^{\infty} u^{k} A_{k}$, then $R\left(u ; T^{*}\right)=$ $\sum_{k=-\infty}^{\infty} u^{k} A_{k}^{*}$ in a punched neighborhood of 0 . Therefore 0 is a pole of $T$ of order $p$ if and only if it is a pole of $T^{*}$ of order $p$, hence $s_{11}(T)=s_{11}\left(T^{*}\right)$, by [7, Theorem 2.1]. It is known that $R(T)$ and $R\left(T^{*}\right)$ are closed simultaneously, and if they are, then $n\left(T^{*}\right)=$ $d(T)$ and $d\left(T^{*}\right)=n(T)$. Hence we obtain the general statements for the indices $i=2,3,4$, $7,8,9,10$. If $T$ is prespectral, so is $T^{*}$ by $[1,3.10]$; thus the last statement follows from the Lemma. 
Remark. For an arbitrary $T$, in $B(X)$, it is known that $s_{5}(T) \supset s_{6}\left(T^{*}\right)$ and $s_{6}(T) \supset$ $s_{5}\left(T^{*}\right)$, where the inclusions can be proper; cf. Pietsch [9, pp. 363-367].

According to Taylor [10, pp. 235-236], we say that an operator $T$, in $B(X)$, is in state I, II or III, if $R(T)$ is $X$, dense in $X$ but not equal to $X$, or not dense in $X$, respectively. Further, $T$ is in state 1,2 or 3 , according as $T^{-1}$ exists and is continuous, exists but is not continuous, or does not exist, respectively. $T$ is in state $A_{b}$, if it is in the states $A$ and $b$ ( $A=$ I, II, III; $b=1,2,3)$, and we say that $T$, or that the pair $\left(T, T^{*}\right)$, is in the state $\left(A_{b}\right.$, $C_{d}$ ), if $T$ is in state $A_{b}$ and $T^{*}$ is in state $C_{d}$.

THEOREM 4. (i) If $T$, in $B(X)$, is prespectral, then $T$ is not in the states $\left(\mathrm{I}_{3}, \mathrm{III}_{1}\right)$ or $\left(\mathrm{III}_{1}\right.$, $\left.\mathrm{I}_{3}\right)$. (ii) If $T$ is prespectral of finite type, then the states $\left(\mathrm{II}_{3}, \mathrm{III}_{2}\right)$ and $\left(\mathrm{III}_{2}, \mathrm{II}_{3}\right)$ are impossible. (iii) If $T$ is spectral of finite type, then the state $\left(\mathrm{III}_{2}, \mathrm{III}_{3}\right)$, and if $T$ is prespectral of finite type and $X^{*}$ is weakly complete, then the state $\left(\mathrm{II}_{2}, \mathrm{III}_{2}\right)$ are impossible. (iv) All other states, possible by the state diagram in [10, p. 237], can actually occur for spectral (if excluded by (iii), then prespectral) operators.

Proof. If $T$ is prespectral, so is $T^{*}$ by $[1,3.10]$. If $T$ is in state 1 , then for every $x$ in $X$ and some positive $p$ we have $|T x| \geqslant p|x|$; hence $R(T)=X$ by [2, Theorem 1], which proves (i). If $T$ is prespectral of finite type, with canonical decomposition $T=S+N$ and resolution of the identity $E$ of class $\Gamma$, and $T$ is in state 3 , then $E_{0} \neq 0$ by [4, Theorem 6.13]. $T\left|E_{0} X=N\right| E_{0} X=N_{0}$ is nilpotent; hence either $N_{0}=0$ or $N_{0}^{k} \neq 0$ and $N_{0}^{k+1}=0$, for some positive integer $k$. We show that even in the latter case $R\left(N_{0}\right)$ is not dense in $E_{0} X$. Supposing it is, we can choose elements $x, x_{n}(n=1,2, \ldots)$ in $E_{0} X$ such that $N_{0}^{k} x \neq 0$ and $N_{0} x_{n} \rightarrow x$. But then $0=N_{0}^{k+1} x_{n} \rightarrow N_{0}^{k} x$, a contradiction. By [10, Theorem 5.4-B], $R(T)$ is not dense in $X$; hence the state $\mathrm{II}_{3}$ is impossible for $T$. Since $T^{*}$ is also prespectral of finite type, (ii) is proved. Finally, if $T$ is prespectral of finite type and $X^{*}$ is weakly complete, then $T^{*}$ is spectral of finite type. For such operators the residual spectrum is empty, by [5, XV. 8.3] which proves (iii).

The states $\left(\mathrm{I}_{1}, \mathrm{I}_{1}\right),\left(\mathrm{II}_{2}, \mathrm{II}_{2}\right)$ and $\left(\mathrm{III}_{3}, \mathrm{III}_{3}\right)$ can occur even for a bounded selfadjoint operator in Hilbert space. To complete the proof we give the following examples.

Example 1. Let $X=\ell_{1}$ and for $x=\left(x_{1}, x_{2}, \ldots\right) \in \ell_{1}$ let $T x=\left(c_{1} x_{1}, c_{2} x_{2}, \ldots\right)$ with $c_{k}=k^{-1}$. Define $F_{k} x=\left(0, \ldots, 0, x_{k}, 0, \ldots\right)$ and $E(b) x=\sum_{c_{k} \in b} F_{k} x$ (b Borel set). Then $T$ is spectral of scalar type with resolution of the identity $E$, and $T$ is in state $\mathrm{II}_{2}$. In $X^{*}=\ell_{\infty}$ for $y^{*}=\left(y_{1}, y_{2}, \ldots\right)$ we have $T^{*} y^{*}=\left(c_{1} y_{1}, c_{2} y_{2}, \ldots\right)$, and $T^{*}$ is in state III $_{2} . T^{*}$ is prespectral of scalar type with resolution of the identity $E^{*}$ of class $X$, but not spectral. By (ii) and [10, p. 237] $T^{* *}$, prespectral of scalar type of class $X^{*}$, must be in state $\mathrm{III}_{3}$.

EXAMPLE 2. The states $\left(\mathrm{III}_{2}, \mathrm{III}_{3}\right)$ and $\left(\mathrm{II}_{2}, \mathrm{III}_{2}\right)$ are possible for quasinilpotent operators in $B(X)$, even if $X^{*}$ is weakly complete. Let $X=C[0,1]$, the space of functions continuous in $[0,1], T x=y$, where $y(t)=\int_{0}^{t} x(s) d s$. Then $T$ is in state $\mathrm{III}_{2}$, and $X^{*}$ is $N B V[0,1]$, the space of functions of bounded variation normalized by the requirements of 
continuity from the right in $(0,1)$ and $x^{*}(0+)=0$. By the Riesz representation theorem,

$$
\left(T^{*} x^{*}\right)(t)=\int_{0}^{t}\left(x^{*}(1)-x^{*}(s)\right) d s ;
$$

hence $R\left(T^{*}\right) \subset A C[0,1]$, the subspace of absolutely continuous functions, and $T^{*}$ is in state $\mathrm{III}_{3}$.

Now let $T_{0}$ denote the restriction of $T$ to $X_{0}=C_{0}[0,1]$, the subspace of functions such that $x(0)=0$; then $T_{0}$ is in state $\mathrm{II}_{2}$. The annihilator of $X_{0}$ in $N B V[0,1]$ is the set of all functions vanishing in $(0,1]$; which are continuous from the right in $[0,1)$. Since $N B V$ is weakly complete, so is $N B V_{0}$, and $T_{0}^{*}$ is in the state $\mathrm{III}_{2}$.

Example 3. The states $\left(\mathrm{III}_{2}, \mathrm{II}_{3}\right)$ and $\left(\mathrm{II}_{3}, \mathrm{III}_{2}\right)$ are possible even for quasinilpotent operators in $B\left(\ell_{2}\right)$. For $x=\left(x_{1}, x_{2}, \ldots\right) \in \ell_{2}$ define $T x=\left(0, a_{1} x_{1}, a_{2} x_{2}, \ldots\right)$, where $a_{k}=2^{-k}$. Then $\left|T^{n}\right| \leqslant a_{1} \ldots a_{n}=2^{-(n+1) n / 2} ;$ thus $T$ is quasinilpotent and is in state $\mathrm{III}_{2}$. For $y^{*}=\left(y_{1}, y_{2}, \ldots\right) \in \ell_{2}$ we have $T^{*} y^{*}=\left(a_{1} y_{2}, a_{2} y_{3}, \ldots\right)$; hence the pair $\left(T, T^{*}\right)$ is in state $\left(\mathrm{III}_{2}, \mathrm{II}_{3}\right)$. Finally, the quasinilpotent $T^{*}$ in $B\left(\ell_{2}\right)$ is clearly in the state $\left(\mathrm{II}_{3}, \mathrm{III}_{2}\right)$.

According to [10, p. 237] and Theorem 4, we obtain the following state diagram.

STATE DIAGRAM FOR PRESPECTRAL OPERATORS

$r$ : Impossible with $X$ reflexive,

$\begin{array}{ccccccc} & \mathrm{III}_{3} & x & x & x & r \text { or } s f & \\ \text { State of } T^{*} & \mathrm{III}_{2} & x & r \text { or } f^{*} & f & x & x \\ & \mathrm{II}_{3} & x & x & x & f & x \\ & \mathrm{II}_{2} & x & & x & x & x \\ & \mathrm{I}_{1} & & x & x & x & x \\ & & \mathrm{I}_{1} & \mathrm{II}_{2} & \mathrm{II}_{3} & \mathrm{III}_{2} & \mathrm{III}_{3}\end{array}$

State of $T$

$f$ : Impossible with $T$ of finite type,

$f^{*}$ : Impossible if $T$ is of finite type and $X^{*}$ is weakly complete,

sf: Impossible with $T$ spectral of finite type.

The states marked by $x$ or not occurring in the diagram are impossible in the general case.

\section{REFERENCES}

1. E. Berkson and H. R. Dowson, Prespectral operators, Illinois J. Math., 13 (1969), 291-315.

2. H. R. Dowson, Restrictions of prespectral operators, J. London Math. Soc., (2) 1 (1969), 633-642.

3. H. R. Dowson, A commutativity theorem for prespectral operators, Illinois J. Math., 17 (1973), 525-532.

4. H. R. Dowson, Some properties of prespectral operators, Proc. Roy. Irish Acad., Sect. A 74 (1974), 207-221.

5. N. Dunford and J. T. Schwartz, Linear operators-Part III: Spectral operators (WileyInterscience, New York, 1971). 
6. B. Gramsch and D. Lay, Spectral mapping theorems for essential spectra, Math. Ann., 192 (1971), 17-32.

7. D. Lay, Spectral analysis using ascent, descent, nullity and defect, Math. Ann., 184 (1970), 197-214.

8. B. Nagy, Essential spectra of spectral operators, to appear.

9. A. Pietsch, Zur Theorie der $\sigma$-Transformationen in lokalkonvexen Vektorräumen, Math. Nachr., 21 (1960), 347-369.

10. A. E. Taylor, Introduction to functional analysis, (Wiley, New York, 1958).

11. A. E. Taylor and C. J. A. Halberg, General theorems about a linear operator and its conjugate, J. Reine Angew. Math., 198 (1957), 93-111.

Department of Mathematics,

TeChNical University

Budapest, Hungary 